\title{
Treatment of Recurrent \& Resistant Dermatomyositis / Polymyositis
}

\author{
Abdel-Azeim M. Alhefny \\ Prof. of Internal Medicine, Rheumatology \& Immunology, Ain Shams University; Egypt
}

\section{INTRODUCTION}

Dermatomyositis (DM) and polymyositis (PM) are two classic forms of inflammatory myopathy. Most patients respond to initial therapy, and some achieve sustained disease control either off all therapy or with low-dose maintenance therapy.

But many patients with DM or PM require intermittent or even continuous therapy:

- Recurrent disease is defined as the occurrence of a disease flare following the achievement of disease control with treatment.

- Resistant disease does not respond sufficiently to the conventional approaches of glucocorticoids plus either azathioprine or methotrexate. In such cases, alternative approaches to remission induction must be considered.

\section{Recurrent Disease}

Complete discontinuation of treatment is unsuccessful in the majority of patients.

For patients who experience disease flares after the achievement of disease control, there are four specific scenarios.

1. For disease flares at $>10 \mathrm{mg}$ /day of prednisone, we suggest the addition of either azathioprine or methotrexate (if not already used) or treatment of the patient as a case of resistant disease (Grade 2C).

With either action, a higher dose of prednisone, generally in the range of $1 \mathrm{mg} / \mathrm{kg}$ per day, will be required to reestablish disease control.

2. For disease flares at $<10 \mathrm{mg} /$ day of prednisone, we suggest 2 options:

- $\quad$ increasing the prednisone to the lowest dose required to reestablish disease control (according to disease severity $20 \mathrm{mg} /$ day $1 \mathrm{mg} / \mathrm{kg}$ per day)

- and/or increasing the azathioprine or methotrexate dose, if this has not been maximized already (Grade 2C).

Correspondence to Abdel-Azeim M. Alhefny, Department of Internal Medicine, Ain Shams University; Egypt. e-mail: alhefny1@gmail.com
Once disease control is restored, we suggest slower tapering than that which was used during the initial course. Some patients are maintained on low-dose prednisone (e.g., 5 $\mathrm{mg} /$ day) for one year or more.

3. For disease flares off prednisone but on a glucocorticoid-sparing drug, There are two options: reinstituting prednisone at the lowest dose required to reestablish disease control

- $\quad$ and/or changing the glucocorticoid-sparing medication

from azathioprine to methotrexate or vice versa (Grade $2 \mathrm{C}$ ).

If the patient has already failed both azathioprine and methotrexate, treatment as a case of resistant disease is appropriate.

4. For flare off all immunosuppressive medication, we suggest reinstituting prednisone with an initial daily dose that varies according to relapse severity (Grade 2C). The minimum starting dose of prednisone is $20 \mathrm{mg} / \mathrm{day}$. In addition, a glucocorticoid-sparing drug should be resumed or started.

\section{Resistant Disease}

Multiple options exist for treating patients who do not respond adequately to glucocorticoids plus either azathioprine or methotrexate; include:

- Rituximab

- Intravenous immune globulin (IVIG)

- Cyclosporine

- Tacrolimus

- Mycophenolate mofetil

- Cyclophosphamide

- Tumor necrosis factor inhibitors

- Combination therapy with azathioprine and methotrexate

Evidence of clinically significant benefit is greatest with rituximab and IVIG if rituximab fails.

\section{Rituximab}

Rituximab targets CD20-positive cells (i.e., Bcell precursors), leading in most patients to the depletion of B cells in the serum within several weeks 
of administration. The trend with the use of rituximab is to employ two 1 gram doses one week apart.

Other regimens for rituximab treatment include: 1 gram every other week for two treatments, and 375 $\mathrm{mg} / \mathrm{m}^{2}$ once weekly times four doses.

Controlled trials involving larger numbers of patients will be required before the full impact of this treatment can be accurately assessed.

The apparent success of rituximab in the PM patients suggests that B cells may be more important in the pathophysiology of PM than previously recognized.

\section{Intravenous immune globulin}

If rituximab is not effective, we suggest IVIG as the second-line agent for the treatment of resistant DM (Grade 2B).

The 2012 American Academy of Neurology guidelines support the use of IVIG for refractory DM but found evidence insufficient to support or refute its use in PM. The expense of this treatment is an important consideration in its long-term use.

Two studies, one in DM and one in PM, have provided evidence that IVIG is an effective short-term therapy for resistant myositis. The mechanism of action is not known.

Prednisone (mean dose $25 \mathrm{mg} /$ day) and a monthly infusion of either IVIG $(2 \mathrm{~g} / \mathrm{kg})$.

Plus one or more additional therapies, including methotrexate, azathioprine, cyclophosphamide, cyclosporine, chlorambucil, plasmapheresis, lymphapheresis, and total body irradiation.

Or patient was treated with IVIG $(1 \mathrm{~g} / \mathrm{kg}$ per day for two days per month for four to six months).

Data related to the use of rituximab and IVIG in interstitial lung disease are extremely limited.

\section{Rituximab versus IVIG}

The reasons for favoring rituximab over IVIG, the only treatment tested in a randomized trial, are the following:

- Rituximab appears to be effective in connective tissue disorders resembling DM and PM, such as systemic lupus erythematosus and rheumatoid arthritis.

- If effective, rituximab may be more likely to lead to a prolonged period of disease control. Many patients who respond to IVIG require continued treatments on a monthly basis.

\section{Calcineurin inhibitors}

Achieve their effects by interfering with $\mathrm{T}$ cell function. The limited evidence available presently suggests that tacrolimus offers some advantage over cyclosporine in efficacy, but larger studies are required before definitive conclusions are possible.

\section{Cyclosporine}

Efficacy for cyclosporine has been suggested for both primary therapy and resistant disease, including interstitial lung disease.

In one report, six patients previously resistant to methotrexate, azathioprine, cyclophosphamide, and/or IVIG underwent treatment with a mean daily cyclosporine dose of $3.5 \mathrm{mg} / \mathrm{kg}$. Over the median six month course of treatment with cyclosporine, the mean daily prednisone dose was reduced by 75 percent. All six patients demonstrated improved strength in the shoulder girdle; four had stronger hip flexor muscles.

\section{Tacrolimus}

Tacrolimus has been used in a limited number of patients with inflammatory myopathy. The optimal dose for this indication is not certain.

In one report, tacrolimus $(0.075 \mathrm{mg} / \mathrm{kg}$ per day in two divided doses) was effective in a series of eight patients with refractory PM complicated by ILD. Strength normalized in five of eight anti-Jo-1 antibody-positive patients and improved in the two anti-SRP positive patients. The mean CK declined from 3114 to $87 \mathrm{IU} / \mathrm{mL}$. Three of five patients with ILD also showed improvement in pulmonary function.

Some data suggest that calcineurin inhibitors are particularly effective in the treatment of ILD complication of DM or PM. For ILD that is refractory to glucocorticoids plus either azathioprine or methotrexate, we use tacrolimus $(0.2 \mathrm{mg} / \mathrm{kg}$ per day in divided doses) as the next agent (Grade 2C).

\section{Mycophenolate mofetil}

In patients with inflammatory myopathy, mycophenolate mofetil ( 1 to $1.5 \mathrm{~g}$ twice daily) is a reasonable alternative if rituximab and IVIG have failed.

Clinicians must be alert to the possibility of opportunistic infection.

\section{Cyclophosphamide}

The patients were treated with IV cyclophosphamide at doses ranging from 300 to 800 $\mathrm{mg} / \mathrm{m}^{2}$ every four weeks plus prednisone. All patients received at least six courses.

Because of their substantial side effect profiles, we suggest reserving alkylating agents (cyclophosphamide and chlorambucil) for patients whose disease has proven resistant to multiple other treatment options (Grade 1C).

\section{Tumor necrosis factor inhibitors}

Data related to the efficacy of tumor necrosis factor inhibition in DM and PM are mixed. Only small studies of etanercept and infliximab have been reported, and further studies will be required to further define their role. The following observations illustrate the range of findings: 
- We suggest not using tumor necrosis factor inhibitors in DM or PM, unless all other treatment options have failed (Grade 2C).

- Combination therapy - with azathioprine (up to $200 \mathrm{mg} /$ day) and methotrexate (up to 25 $\mathrm{mg} /$ week) hold some potential for efficacy in patients with resistant disease. However, the risk of treatment-related morbidity when using both of these medications together mandates the utmost care in monitoring patients for cytopenias and other adverse effects.

\section{Refractory rash}

In some cases of DM, the cutaneous manifestations are more refractory to treatment than the muscle disease.

\section{REFERENCES}

1. Noss EH, Hausner-Sypek DL, Weinblatt ME. Rituximab as therapy for refractory polymyositis and dermatomyositis. J Rheumatol 2006; 33:1021.

2. Mahler EA, Blom M, Voermans NC, et al. Rituximab treatment in patients with refractory inflammatory myopathies. Rheumatology (Oxford) 2011; 50:2206.

3. Patwa HS, Chaudhry V, Katzberg H, et al. Evidencebased guideline: intravenous immunoglobulin in the treatment of neuromuscular disorders: report of the Therapeutics and Technology Assessment Subcommittee of the American Academy of Neurology. Neurology 2012; 78:1009.

4. Cherin P, Pelletier S, Teixeira A, et al. Results and long-term followup of intravenous immunoglobulin infusions in chronic, refractory polymyositis: an open study with thirty-five adult patients. Arthritis Rheum 2002; 46:467.
5. Qushmaq KA, Chalmers A, Esdaile JM. Cyclosporin A in the treatment of refractory adult polymyositis/dermatomyositis: population based experience in 6 patients and literature review. J Rheumatol 2000; 27:2855.

6. Ochi S, Nanki T, Takada $\mathrm{K}$, et al. Favorable outcomes with tacrolimus in two patients with refractory interstitial lung disease associated with polymyositis/dermatomyositis. Clin Exp Rheumatol 2005; 23:707.

7. Rowin J, Amato AA, Deisher N, et al. Mycophenolate mofetil in dermatomyositis: is it safe? Neurology 2006; 66:1245.

8. Pisoni CN, Cuadrado MJ, Khamashta MA, et al. Mycophenolate mofetil treatment in resistant myositis. Rheumatology (Oxford) 2007; 46:516.

9. Sinoway PA, Callen JP. Chlorambucil. An effective corticosteroid-sparing agent for patients with recalcitrant dermatomyositis. Arthritis Rheum 1993; 36:319.

10. Yamasaki Y, Yamada $H$, Yamasaki $M$, et al. Intravenous cyclophosphamide therapy for progressive interstitial pneumonia in patients with polymyositis/ dermatomyositis. Rheumatology (Oxford) 2007; 46: 124.

11. Muscle Study Group. A randomized, pilot trial of etanercept in dermatomyositis. Ann Neurol 2011; 70:427.

12. Anandacoomarasamy A, Howe G, Manolios N Advanced refractory polymyositis responding to infliximab. Rheumatology (Oxford) 2005; 44:562.

13. Efthimiou P, Schwartzman S, Kagen LJ. Possible role for tumour necrosis factor inhibitors in the treatment of resistant dermatomyositis and polymyositis: a retrospective study of eight patients. Ann Rheum Dis 2006; 65: 1233. 